\title{
The histopathological differential diagnosis of gastrointestinal stromal tumours
}

\author{
J F Graadt van Roggen, M L F van Velthuysen, P C W Hogendoorn
}

\begin{abstract}
Gastrointestinal stromal tumours (GISTs), initially presumed to be of "true" smooth muscle origin, encompass a heterogeneous, and as yet incompletely understood, group of mesenchymal tumours with respect to their origin, cellular differentiation, and prognosis. Cellular morphology ranges from predominantly spindle shaped to epithelioid in character, whereas differentiation pathways, as determined primarily by immunohistochemistry and ultrastructure, can vary from indeterminate to myoid and/or neural. Recent work has indicated that the interstitial cells of Cajal, a complex cellular network postulated to act as pace-
\end{abstract} maker cells of the gastrointestinal tract, which exhibit both myoid and neural features, could be candidates for tumour histogenesis. This would provide a plausible and attractive explanation for the variable differentiation pathways identified in the GIST category to date. Nevertheless, the occasional but undisputed location of GISTs outside the gastrointestinal tract (omentum, peritoneum, and retroperitoneum) might mitigate against such an origin, and their histogenesis remains open to debate. The c-kit proto-oncogene, encoding a growth factor receptor with tyrosine kinase activity, has been postulated to play an important role in tumorigenesis because "gain of function" mutations in this gene, localised to chromosome 4q11-21, are being increasingly identified in hereditary and sporadic cases. Monoclonal and polyclonal antibodies directed at the c-kit gene product expressed on the cell surface (CD117/c-kit) appear to be increasingly helpful in resolving the histopathological differential diagnosis between GISTs and true gastrointestinal smooth muscle neoplasms, schwannomas, and other far less frequently occurring mesenchymal tumours at this site. Although tumours with a clinically benign course appear to be more common than their malignant counterparts, no specific histological criteria have as yet been identified to enable an unambiguous prediction of biological behaviour. Increasing tumour size and mitotic activity favour aggressive tumour behaviour, whereas the prognostic value of germline and somatic mutations within the c-kit proto-oncogene remains to be elucidated further. It is the aim of this synopsis to highlight the relevant fundamental and diagnostic developments with respect to this complex group of neoplasms. (f Clin Pathol 2001;54:96-103)
Keywords: gastrointestinal stromal tumours; c-kit; diagnosis

In contrast to first impressions, it is now increasingly apparent that the category of mesenchymal tumours designated "gastrointestinal stromal tumours" (GISTs) encompasses a clinicopathologically distinctive but heterogeneous, and as yet poorly understood, group of neoplasms with respect to their origin, cellular differentiation, and prognosis. ${ }^{12}$

Most gastrointestinal mesenchymal tumours were initially presumed to be of smooth muscle origin, popularly labelled leiomyoma (benign) or leiomyosarcoma (malignant) when composed primarily of spindle shaped cells and benign or malignant leiomyoblastoma when composed primarily of cells with an epithelioid morphology. ${ }^{3-5}$ However, further studies have demonstrated that within the GIST group there appears to be considerable variability in cellular differentiation at a morphological, immunohistochemical, and ultrastructural level. This phenotypic variability ranges from indeterminate ("uncommitted") to incompletely myoid and/or neural when compared with the characteristic features of true smooth muscle tumours occurring at this site and elsewhere in the body (table 1). ${ }^{6-19}$ The above mentioned features, together with the increasing understanding of the (molecular) genetic changes identified within the GIST category, provide sufficient clinicopathological evidence to validate their distinction from "classic"/true gastrointestinal smooth muscle tumours. ${ }^{60}$

Furthermore, in contrast to typical smooth muscle tumours, it has become apparent that the variability in natural biological behaviour of these tumours is not predictable histopathologically using the generally accepted criteria associated with malignant potential. The numerous acronyms used for these neoplasmswhich include STUMP (smooth muscle tumour of uncertain malignant potential), GIST (gastrointestinal stromal tumour), GANT (gastrointestinal autonomic nerve tumour), and most recently GIPACT (gastrointestinal pacemaker cell tumour) - clearly attest to the uncertainty and controversy concerning the histogenesis and clinical course of these tumours. ${ }^{12618}$

Recent work is beginning to indicate that the interstitial cells of Cajal (ICC) (or more probably a precursor stem cell) of the gastrointestinal tract, which are believed to play an important function in the control of intestinal motility, might represent a possible histogenetic origin for the GIST category. ${ }^{18} 2122$ GISTs share numerous morphological, immunohistochemical, and ultrastructural features with the 
Table 1 Morphological comparison of smooth muscle neoplasms and gastrointestinal stromal tumours (GISTs)

\begin{tabular}{lll}
\hline & Smooth muscle tumours & GIST \\
\hline Mic & Variably spindled cells & Variable morphology \\
& Cigar shaped nuclei & Often more spindly nuclei \\
& Eosinophilic cytoplasm & Variably eosinophilic \\
& Bipolar, perinuclear location of cytoplasmic glycogen & Variable cytoplasmic glycogen \\
IHC & Vimentin positive & Vimentin positive \\
& SMA, MSA generally positive & SMA, MSA patchylabsent \\
& Desmin frequently positive (50-70\% cases) & Desmin usually negative \\
& CD34 may be focally positive & CD34 often positive $\mathbf{5 0 - 8 0 \%}$ of cases)
\end{tabular}

Relevant features are highlighted in bold.

EM, electron microscopy; IHC, immunohistochemistry; Mic, microscopy; MSA, muscle specific actin; NSE, neurone specific enolase; SMA, smooth muscle actin.

ICCs, ${ }^{23-25}$ providing a plausible explanation for the variable differentiation identified in GISTs to date. Nevertheless, the non-gastrointestinal location (omentum, peritoneum, and retroperitoneum $)^{26}$ of a small proportion of cases indicates that the issue of histogenesis is still uncertain.

In this review we discuss the more recent and relevant fundamental and diagnostic developments with respect to this category of tumours.

Current definition and epidemiology

GISTs account for most mesenchymal tumours arising within the gastrointestinal tract. ${ }^{126}$ Although this generally accepted and popular designation highlights the non-epithelial nature of gastrointestinal stromal tumours, it nevertheless represents a specific category of, as yet, incompletely characterised benign and malignant neoplasms with an incomplete myogenic and/or neural or "uncommitted" phenotype primarily at the immunohistochemical and ultrastructural level. Tumours of true smooth muscle, neural (schwannian), fibroblastic, and vascular origin are excluded.

The incidence of clinically malignant GISTs based on data from the Finish Cancer Registry is roughly four/million inhabitants in southern Finland, ${ }^{2}$ although in our experience this is probably a conservative estimate; the ratio of benign/malignant GISTs remains difficult to measure because of the lack of unequivocal histopathological criteria for predicting tumour behaviour. GISTs arise most commonly within the wall of the stomach $(65-70 \%)$ and small intestine $(30-45 \%)$, and are seen far less frequently in the oesophagus, colon, and rectum, where true myogenic tumours predominate. ${ }^{12}$ They generally present in adults with a peak incidence during the fifth and sixth decades, being infrequent before the age of 40 , and no significant sex difference has been noted. ${ }^{12}$ Aetiological factors have not been identified; a putative association with cellular Epstein-Barr virus infection has not been substantiated. ${ }^{27-29}$ Clinical signs and symptoms (nausea, vomiting, abdominal pain, anaemia, and melaena) are non-specific and consequently not helpful for (differential) diagnostic purposes.

\section{Histomorphology}

Grossly, tumours vary greatly in size, ranging from $1-2 \mathrm{~cm}$ to more than $20 \mathrm{~cm}$ in diameter. The tumours are usually well circumscribed and generally unencapsulated, although a pseudocapsule may occasionally be seen. The lesions are either submucosal (with or without ulceration of the overlying mucosa), intramural, or subserosal. On sectioning, the cut surface varies in colour from grey/white to red/ brown, depending on the degree of haemorrhage, and may be solid, partially cystic, or necrotic. Within the GIST category, cellular features demonstrate a broad morphological spectrum but there are two principal histological patterns: a spindle cell $(60-70 \%$ of cases) (fig 1B) or epithelioid (30-40\% of cases) (fig 1A) character, or a combination of both in variable proportions.

Tumours composed primarily of spindle shaped cells are generally compact and highly cellular with a patternless, fascicular, whorled, storiform, or palisading architecture and minimal tumour stroma; epithelioid tumours may have a more fascicular or "nested" appearance, although all the other architectural patterns mentioned above may be identified. Within tumours composed primarily of spindle shaped cells, the cytoplasm may be either eosinophilic, basophilic, or amphophilic with a somewhat fibrillar appearance. Within the epithelioid group, the cytoplasm is more abundant, ranging from amphophilic to clear, and cellular borders are more clearly defined; a predominantly oncocytic cytoplasmic character has been described recently. ${ }^{30}$ Cytoplasmic glycogen with a perinuclear distribution is regularly present. In general, the nuclear features of GISTs are highly variable, ranging from a monotonous predominantly oval/spindly appearance to obviously pleomorphic; they contain nucleoli of variable prominence and multinucleation may be seen but is not a prominent feature. Mitotic activity may be virtually absent or high. A variable inflammatory infiltrate composed mainly of lymphocytes and plasma cells may be seen. Haemorrhage and necrosis may be present. The prominence of a vascular network is variable. 

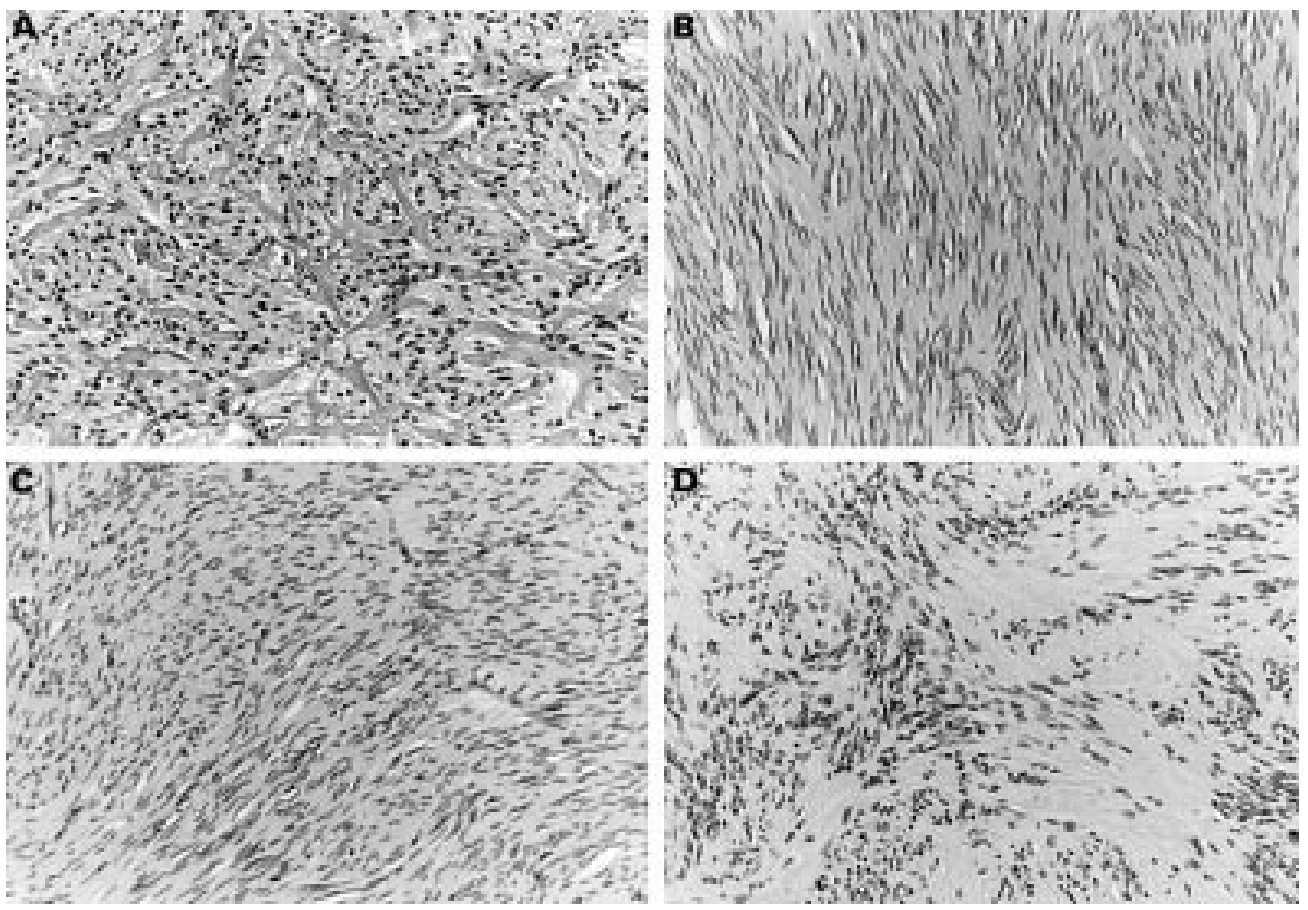

Figure 1 Medium power view (magnification, $\times 200$; haematoxylin and eosin stain) illustrating the typical histological features of an (A) epithelioid and (B) spindle cell GIST, (C) a gastrointestinal leiomyoma (oesophagus), and (D) a schwannoma. It is evident that on morphological grounds alone this differential diagnosis may be extremely problematic, and the associated clinical implications may be considerable.

This broad histomorphological profile, together with the immunohistochemical and ultrastructural evidence, suggests a diversity of differentiation options detailed below (table 1).

\section{Immunohistochemistry}

Review of the literature with respect to the immunohistochemistry of GISTs yields a diverse and confusing array of data. ${ }^{3-17} 19$ 31-33 This lack of unanimity may be related to case selection. Because of the uncertainty about the histopathological criteria for categorising an intestinal mesenchymal tumour to the GIST group, and in particular their distinction from true smooth muscle tumours, it is probable that the different reported studies represent analyses of heterogeneous tumour populations. An increasingly frequent correlation of the cellular morphology with the immunohistochemical profile, ultrastructural features and, more recently, molecular genetic findings, and the subsequent removal of tumours with complete smooth muscle differentiation from the GIST category (leiomyoma/leiomyosarcoma), has sharpened the immunohistochemical picture of GISTs to some extent. An additional reason for the reported diversity could be related to the wide choice of monoclonal and polyclonal antibodies available (with their varying specificities), as well as differences in the technical parameters used and variable interpretation of the staining results by the individual pathologists involved.

However, despite these possible variables, the antibodies most commonly used in the various studies to characterise GISTs are those directed against vimentin, desmin, muscle specific actin (MSA/HHF-35), smooth muscle actin (SMA), S100 protein, neurofilament (NF), neurone specific enolase (NSE), PGP9.5, CD34, and CD117 (c-kit).

With respect to the controversy surrounding a myogenic origin, the myoid markers MSA and SMA are variably expressed, whereas desmin is almost never present. Furthermore, MSA and SMA are generally only focally present or completely absent, indicating that at most smooth muscle differentiation is probably incomplete (table 1). ${ }^{126}$

A possible schwannian/neural differentiation (S100 protein, PGP9.5, and NSE positivity) may be present in a proportion of SMA and MSA negative tumours; a small proportion of tumours express both myogenic and schwannian/neural differentiation, whereas a small number of GISTs are positive for vimentin only and exhibit no detectable differentiation at an immunohistochemical level. Nevertheless, as is evident from the above data, the nature of this diversity remains a controversial area requiring further study.

Antibodies to CD34 and CD117 in particular, although not tumour specific, are generally used in the differential diagnosis of GISTs from smooth muscle and other intestinal mesenchymal tumours because they appear to be expressed in most GISTs. ${ }^{1-18}$ 31-37

CD34 (MY10, QBEND10) is a $110 \mathrm{kDa}$ transmembrane glycoprotein present on human haematopoietic progenitor cells and vascular endothelium. ${ }^{38} \mathrm{CD} 34$ is expressed by a wide variety of tumours (table 2) and is detectable in $50-80 \%$ of GISTs (table 3 ). However, because CD34 may also be expressed by true smooth muscle cells, it is not a particularly helpful marker on its own. Nevertheless, in combination with CD117 and 
Table 2 CD34 positive tumours

Gastrointestinal tumours

- Gastrointestinal stromal tumours

Tumours occurring primarily outside the gastrointestinal tract

- Non-gastrointestinal smooth muscle tumours

- Solitary fibrous tumour

- Dermatofibrosarcoma protuberans

- Kaposi's sarcoma

- Lipoma/angioliopma/spindle cell lipoma/atypical lipoma

- Neurofibroma

- Vascular tumours

- Epithelioid sarcoma

Although CD34 positivity, as part of an appropriate immunohistochemical panel, is diagnostically useful in the context of gastrointestinal spindle cell tumours, it is important to realise that it is a ubiquitous antigen present in many different tumours.

Table 3 Immunohistochemical positivity for CD34 and CD117 in gastrointestinal stromal tumours (GISTs)

\begin{tabular}{lll}
\hline CD117 & CD34 & Ref \\
\hline $100 \%(78 / 78)$ & $72 \%(56 / 78)$ & 18 \\
$94 \%(46 / 49)$ & $82 \%(42 / 49)$ & 39 \\
$88 \%(28 / 32)$ & $69 \%(22 / 32)$ & 18 \\
$81 \%(69 / 85)$ & $56 \%(48 / 85)$ & 34
\end{tabular}

CD117 is the more sensitive marker and CD117 might not be positive in all GISTs.

S100 it can still be useful in differentiating between GIST and other mesenchymal tumours encountered in the intestinal tract. ${ }^{18} 3439$

Far more useful than CD34 is CD117/c-kit, discussed in more detail later. Once again, although not cell or tumour specific (tables 4 and 5), CD117 is expressed in $80-100 \%$ of GISTs (table 3), irrespective of tumour cytomorphology. Furthermore, CD117 is not expressed in smooth muscle or neural tumours and is consequently a powerful aid in the differential diagnosis between GISTs and other gastrointestinal mesenchymal tumours. ${ }^{18} 3439 \mathrm{~A}$ recent clinicopathological study of GISTs primary in the omentum, peritoneum, and retroperitoneum has reinforced the importance of CD117 in making the distinction between GISTs at unusual locations and morphologically comparable mesenchymal tumours at these sites, facilitating a correct diagnosis and appropriate clinical follow up. ${ }^{26}$

\section{Ultrastructural features}

Electron microscopy has provided some degree of clarification of the range of cellular differentiation in the GIST group. ${ }^{126141618193140}$ In contrast to the ultrastructural features of true smooth muscle cells, there is clearly a wide spectrum of cellular differentiation within and between GISTs (table 1). Well developed myo-

Table 4 Normal tissues expressing CD117/c-kit (adapted from Chan ${ }^{1}$ )

\begin{tabular}{ll}
\hline Gastrointestinal tract & Sites outside gastrointestinal tract \\
\hline Interstitial cells of Cajal & A subset of CD34 positive haemopoietic stem cells \\
Mast cells & Melanocytes \\
& Basal cells epidermis \\
& Immature Langerhans cells in the epidermis \\
& Variety of epithelial cells (breast/salivary gland/sweat gland/renal \\
& tubule) \\
& Cells present in the reproductive system \\
& A subset of glial cells \\
& Osteoclast precursor
\end{tabular}

Tabulation of the diverse cell types that might express CD117/c-kit. Consequently, although CD117 is a diagnostically useful antigen expressed by the interstitial cells of Cajal (and in most gastrointestinal stromal tumours), it is important to be aware of the expression of this antigen in a variety of other cells.
Table 5 CD117/c-kit positive tumours (adapted from Chan ${ }^{1}$ )

Gastrointestinal tumours

- Gastrointestinal stromal tumours

Tumours occurring primarily outside the gastrointestinal tract

- Melanoma (loss in vertical growth phase and metastases)

- Clear cell sarcoma of tendons and aponeuroses

- Endometrial carcinoma

- Anaplastic small cell carcinoma of the lung

- Ewing's sarcoma group

- Anaplastic large cell lymphoma

- Reed-Sternberg cell in Hodgkin's lymphoma

- Mastocytosis

- Acute myeloid leukaemia

- Glioma

- Germinoma

CD117/c-kit positivity, in the setting of an appropriate immunohistochemical panel, is very useful in resolving the differential diagnosis of gastrointestinal spindle cell tumours. Nevertheless, it is important to remember that this antigen is present in a variety of different tumours, particularly in the context of metastatic disease.

filaments are not often present, as is the case for well developed focal densities and attachment plaques. Nevertheless, focal bundles of actin myofilaments and inconspicuous dense bodies are seen in as many as $40 \%$ of cases and correlate with the immunohistochemical evidence of incomplete myoid differentiation. ${ }^{214}$ In addition, complex cytoplasmic extensions and neurite-like processes, microtubules, synapselike structures, and dense core granules indicative of variable neural differentiation, which correlate with the immunohistochemically observed nerve sheath/neural phenotype, are seen independently in a proportion of cases and in combination with the above mentioned myoid features. ${ }^{12614}$ Skeinoid fibres, representing extracellular amorphous eosinophilic arrays of interwoven modified collagen, may occasionally be seen and are an additional feature indicative of neural differentiation. ${ }^{14}$ In contrast to the above mentioned subgroups, a small proportion of cases exhibit no diagnostic features and are referred to as being of uncommitted type. ${ }^{14}$ Considering the immunohistochemical expression of CD34 and CD117 in most GIST subgroups, including the uncommitted group, together with the broad spectrum of ultrastructural features seen in these tumours, the increasingly accepted hypothesis is that the GIST group might represent a variable differentiation spectrum arising from a common precursor cell-discussed belowand that a distinction between the various subtypes is probably artificial. ${ }^{1}$

\section{Molecular biology}

The c-kit proto-oncogene has been mapped to chromosome 4q11-21 (W locus) and encodes a type III tyrosine kinase growth factor receptor belonging to the immunoglobulin supergene family. ${ }^{35} 42$ The c-kit molecule has a molecular weight of $135 \mathrm{kDa}$ and consists of 976 amino acids, incorporating an extracellular domain composed of five immunoglobulin-like regions, a transmembrane domain, and an intracellular domain responsible for the kinase activity. Stem cell factor (kit-ligand/steel factor/ mast cell growth factor) serves as the extracellular receptor ligand and is believed to play a role in cellular survival, proliferation, and differentiation. ${ }^{43}$ The c-kit gene product (c-kit/ 
Table 6 Comparison of criteria reported in the literature to predict biological tumour behaviour

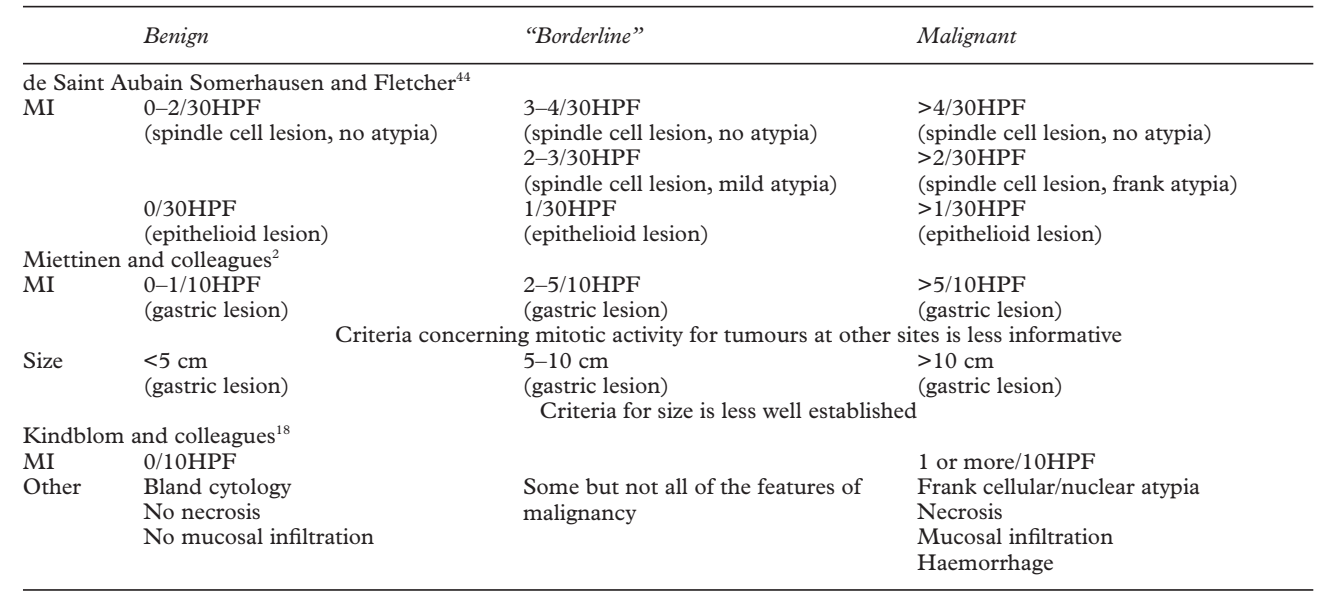

Comparison of the criteria used in attempts to predict biological tumour behaviour. The data highlight the current variability in parameters used as reported in the literature. Although mitotic activity defined in $\mathrm{mm}^{2}$ or field diameter would be preferable, the designation "high power fields (HPF)" is used in the cited literature.

MI, mitotic index.

CD117) is expressed in a wide variety of normal tissues, including the ICCs of the gastrointestinal tract (table 6), ${ }^{43}$ and increasingly germline and sporadic gain of function mutations within c-kit are being detected in GISTs, although their specific role in tumorigenesis remains elusive. ${ }^{20}{ }^{39} 46-48$ Furthermore, partial DNA losses of chromosome 14q (possibly suggesting loci for additional tumour suppressor genes involved in GIST tumorigenesis) have been identified in both clinically benign and malignant GISTs, whereas they have not been identified in leiomyomas or leiomyosarcomas, and might be potential markers for this tumour group. ${ }^{49-51}$

The nature and prognostic relevance of c-kit mutations is very topical and receiving increasing attention in the literature. The identification of germline mutations in families with GISTs suggests an association between genetic aberrations at the c-kit gene locus and the development of GIST. Furthermore, with the increasing identification and clinicopathological documentation of c-kit mutations at multiple sites within the gene, possible associations with prognosis are beginning to be analysed. Although data are still very limited, several recent papers have reported contradictory findings with respect to a possible association between mutation positive GISTs and prognosis. $^{485253}$

\section{Histogenesis}

For a variety of sarcomas, such as synovial sarcoma and epithelioid sarcoma, no benign counterpart has been identified to date, and a histogenetic source remains to be determined. Similarly, the broad morphological spectrum exhibited by GISTs at a light microscopic and ultrastructural level has generated much debate and controversy concerning tumour histogenesis. Although this will probably remain for the foreseeable future, Kindblom et al, ${ }^{18}$ and more recently Sircar et $a l,{ }^{22}$ have elegantly provided considerable support for a possible histogenetic origin from the ICCs encountered in the gastrointestinal tract, which are thought to play a role in coordinating intestinal motility $^{21}{ }^{54}$ (summarised by Chan ${ }^{1}$ ). The ICCs appear to be modified smooth muscle cells occurring at various intramural sites within the intestinal tract, primarily in the muscularis propria and in association with the myenteric plexus. In depth analysis and comparison of these cells with the cellular component of GISTs identified many important similarities: ICCs demonstrate an incomplete myogenic and neural differentiation at an immunohistochemical and ultrastructural level (providing an attractive explanation for the variable differentiation noted in the GIST spectrum, including the GANTs), and exhibit features that can be identified in all the tumour types currently ascribed to the GIST group. In particular, immunohistochemical expression of CD34 and CD117, although not tumour or tissue specific (tables 1-3 and 6), appears to be an important unifying parameter in GISTs. Furthermore, regional variation in the distribution of ICCs in the gastrointestinal tract (being more common in the stomach and the small intestine and least frequent in the oesophagus and rectum) correlates well with the observed prevalence of GISTs at the various anatomical sites. $^{12}$ Their convincing arguments that tumour morphology could be accounted for by variable degrees of differentiation (by mechanisms as yet unknown) in an ICC precursor cell is worth investigating further to elucidate the histogenesis of these interesting but complex neoplasms. Nevertheless, the occasional location of GISTs outside the gastrointestinal tract $^{26}$ (omentum, peritoneum, and retroperitoneum), where the ICCs are not known to be present, indicates that the issue of histogenesis is by no means resolved.

\section{Biological behaviour}

Predicting the potential biological behaviour of these tumours remains difficult and an analysis of the literature to resolve this issue provides many conflicting data. ${ }^{12} 1354-70$ Mitotic activity $^{12} 6135456-586166$ 69-72 tumour size, ${ }^{57636669-72}$ tumour necrosis, ${ }^{547}$ histological type/pattern, ${ }^{67}$ 
immunohistochemical profile, staining for proliferation antigens, and ploidy status, ${ }^{13} 565760-$ 636568 among others, have all been extensively evaluated in this context without any consensus being established. ${ }^{2254}$ An analysis of GISTs at each anatomical site $1265458596669-72$ in an attempt to identify possible site specific prognostic factors has not appreciably facilitated matters, although oesophageal tumours as a group have the most favourable long term survival and small intestinal tumours have the worst. ${ }^{55}$ Although no unequivocal data are as yet available, current efforts are being focused on attempting to establish whether the presence/absence of c-kit mutations, and/or the nature of the mutation, have a bearing on prognosis.

Current data indicate that increasing mitotic activity ${ }^{546366}$ and increasing tumour size $\mathrm{s}^{57-596366}$ may be of some use in predicting biological behaviour, and various cut off values for mitotic activity and tumour size have been proposed (table 6). ${ }^{12}{ }^{22}$ Nevertheless, no individual factor is of unequivocal independent prognostic use, and a constellation of parameters is used to provide some indication of a probable clinical course. As a consequence of the general confusion regarding the natural behaviour of this group of tumours, it is our practice to be extremely cautious with any form of prediction (for example, in histologically bland tumours of large size) and to emphasise the need for stringent longer term clinical follow up.

Aggressive disease, despite surgery with appropriate free resection margins, is characterised by local recurrence, omental and peritoneal seeding, ${ }^{73}$ and metastatic disease to the liver; pulmonary and osseous metastases occur less frequently and primarily in more advanced disease. The value of adjuvant radiotherapy and chemotherapy remains to be determined unequivocally. ${ }^{2}$

Differential diagnostic considerations GISTs, gastrointestinal leiomyoma or leiomyosarcoma, schwannoma, local extension by a primary retroperitoneal dedifferentiated liposarcoma, benign and malignant vascular tumours, intra-abdominal fibromatosis (desmoid tumour), carcinoid with a spindle cell morphology, and metastatic disease (spindle cell melanoma/spindle cell carcinoma) are the predominant tumours that may need to be considered in the differential diagnosis. Most of these tumours can be characterised accurately on the basis of precise clinical data and diligent microscopy, supplemented by appropriate immunohistochemical, ultrastructural, and molecular biological analyses (table 1). Separating GISTs from true smooth muscle tumours, clinically relevant because of differences in biological behaviour, can sometimes be difficult. Although immunohistochemical (CD117 positivity in GISTs) and ultrastructural examination (at most incomplete smooth muscle differentiation in GISTs) should facilitate the distinction from true smooth muscle tumours, it is possible that the rare CD117 (c-kit) negative GIST exhibiting extreme myoid differentiation might not be identifiable with current techniques. The clinically relevant distinction between an S100 positive, CD117/ CD34 negative GIST with a predominantly epithelioid morphology and metastatic melanoma, in the absence of a known primary, could in rare circumstances be potentially problematic; the nature of the $\mathrm{S} 100$ positivity, diffuse in melanoma and at most patchy in GIST, should facilitate this distinction.

\section{Conclusion}

GISTs are the most common mesenchymal neoplasms of the stomach and small intestine and are relatively less frequent at other gastrointestinal sites. A lack of awareness of their broad morphological spectrum can complicate diagnosis. Nevertheless, an increasing awareness of their immunophenotypic, ultrastructural, and genotypic features coupled with an evolving understanding of their histogenesis is facilitating our ability to identify these tumours. Consequently, it should now become increasingly possible (and important) to study selected tumour populations (or subgroups), retrospectively and prospectively, in an attempt to highlight the parameters influencing their biological behaviour.

1 Chan JKC. Mesenchymal tumors of the gastrointestinal tract: a paradise for acronyms (STUMP, GIST, GANT, and now GIPACT). Implications of c-kit in genesis, and yet another of many emerging roles of the interstitial cell of Cajal in the pathogen Anat Pathol 1999;6:19-40.

2 Miettinen M, Sarlomo-Rikala M, Lasota J. Gastrointestinal stromal tumours. Annales Chirurgiae et Gynaecologiae 1998; 87romal tum

3 Golden T, Stout AP. Smooth muscle tumors of the gastrointestinal tract and retroperitoneal tissues. Surg Gynecol Obstet 1941;73:784-810

4 Appelman HD, Helwig EB. Gastric epithelioid leiomyoma and leiomyosarcoma (leiomyoblastoma). Cancer 1976;38: 709-28.

5 Appelman HD, Helwig EB. Sarcomas of the stomach. Am $\mathcal{F}$ Clin Pathol 1977;67:2-10.

6 Appelman HD, Goldman H, Appelman HD, et al, eds. Mesenchymal tumors of the gut: historical perspectives, new approaches, new results, and does it make any difference? In: Gastrointestinal pathology. Baltimore: Williams and Wilkins, 1990:220-46.

7 Hjermsted BM, Sobin LH, Helwig EB. Stromal tumors of the gastrointestinal tract: myogenic or neurogenic? [published erratum appears in Am $\mathcal{f}$ Surg Pathol 1987;11:660].
listron Am f Surg Pathol 1987;11:383-6.

8 Pike AM, Lloyd RV, Appelman HD. Cell markers in gastrointestinal stromal tumors. Hum Pathol 1988;19:830-4.

intestinal stromal tumors. Hum Pathol 1988;19:830-4.
Mazur MT, Clark HB. Gastric stromal tumors, reappraisal of histogenesis. Am f Surg Pathol 1983;7:507-19.

10 Suster S. Gastrointestinal stromal tumors. Semin Diagn Pathol 1996;13:297-313.

$1 \mathrm{Ma}$ CK, Amin MB, Kintanar E, et al. Immunohistologic characterisation of gastrointestinal stromal tumors: a study of 82 cases compared with 11 cases of leiomyomas. Mod Pathol 1993;6:139-44

12 Saul SH, Rast ML, Brooks JJ. The immunohistochemistry of gastrointestinal stromal tumors. Evidence supporting an origin from smooth muscle. Am f Surg Pathol 1987;11:46473 .

13 Ueyama T, Guo KJ, Hashimoto $\mathrm{H}$, et al. A clinicopathologic and immunohistochemical study of gastrointestinal stromal and immunohistochemical study of

14 Erlandson RA, Klimstra DS, Woodruff JM. Subclassification of gastrointestinal stromal tumors based on evaluation by electron microscopy and immunohistochemistry. Ultrastruct Pathol 1996;20:373-93.

15 Hurlimann J, Gardiol D. Gastrointestinal stromal tumors. An immunohistochemical study of 165 cases. Histopathology 1991;19:311-20.

16 Mackay B, Ro J, Floyd C, et al. Ultrastructural observations on smooth muscle tumors. Ultrastruct Pathol 1987;11:593607.

17 Miettinen M. Gastrointestinal stromal tumors. An immunohistochemical study of cellular differentiation. Am $\mathcal{F}$ Clin Pathol 1988;89:601-10.

18 Kindblom L-G, Remotti H, Aldenborg F, et al. Gastrointestinal pacemaker cell tumor (GIPACT). Gastrointestinal stromal tumors show phenotypic characteristics of the interstitial cells of Cajal. Am f Clin Pathol 1998;152: of the interst 
19 Shanks JH, Harris M, Banerjee SS, et al. Gastrointestinal autonomic autonomic nerve tumours: a
Histopathology 1996;29:111-21.

20 Lux ML, Rubin BP, Biase TL, et al. KIT extracellular and kinase kinase domain mutations in gast
tumors. Am $\mathcal{F}$ Pathol 2000;156:791-5.

21 Thuneberg L. Interstitial cells of Cajal: intestinal pacemaker cells? Adv Anat Embryol Cell Biol 1982;71:1-130.

22 Sircar K, Hewlett BR, Huizinga JD, et al. Interstitial cells of Cajal as precursors of gastrointestinal stromal tumors. $A m$ f Surg Pathol 1999;23:377-89.

23 Rumessen JJ, Mikkelsen HB, Thuneberg L. Ultrastructure of interstitial cells of Cajal associated with deep muscular plexus of human small intestine. Gastroenterology 1992;102 $56-68$.

24 Rumessen JJ, Thuneberg L. Interstitial cells of Cajal in human small intestine. Ultrastructural identification and organisation between the main smooth muscle layers. Gastroenterology 1991;100:1417-31.

25 Rumessen JJ, Peters S, Thuneberg L. Light- and electron microscopical studies of interstitial cells of Cajal and muscle cells at the submucosal border of human colon. $L a b$ Invest 1993;68:481-95.

26 Miettinen M, Monihan JM, Sarlomo-Rikala M, et al. Gastrointestinal stromal tumors/smooth mucle tumors (GISTS) primary in the omentum and mesentery: clinicopathologic and immunohistochemical study of 26 cases. Am F Surg Pathol 1999;23:1109-18.

27 van Gelder T, Vuzevski VD, Weiman W. EBV in smoothmuscle tumors. N Engl f Med 1995;332:1719

28 Liebowitz D. Epstein-Barr virus-an old dog with new tricks. N Engl f Med 1995;332:55-7.

29 Kubben FJGM, Kroon FP, Hogendoorn PCW, et al. Absence of Epstein-Barr virus in a gastrointestinal stromal cell tumour (GIST) in an adult human immunodeficiency virus-seropositive patient with past Epstein-Barr virus (EBV) infection. Eur f Gastroenterol Hepatol 1997;9:721-4.

30 Damiani S, Pasquinelli G, Eusebi V. GANT-like gastrointestinal pacemaker cell tumours with oncocytic features. intestinal pacemaker cell tumours with oncocytic features.

31 Lauwers GY, Erlandson RA, Casper ES, et al. Gastrointestinal autonomic nerve tumors. A clinicopathological, immunohistochemical, and ultrastructural study of 12 cases. Am $\mathcal{F}$ Surg Pathol 1993;17:887-97.

32 Matsumoto K, Min W, Yamada N, et al. Gastrointestinal autonomic nerve tumors: immunohistochemical and ultrastructural studies in cases of gastrointestinal stromal tumor. Pathol Int 1997;47:308-14.

33 Dhimes P, Lopez-Carreira M, Ortega-Serrano MP, et al. Gastrointestinal autonomic nerve tumours and their separation from other gastrointestinal stromal tumours: an ultrastructural and immunohistochemical study of seven cases. Virchows Arch A Anat Pathol Histopathol 1995;426: 27-35.

34 Sarlomo-Rikala M, Kovatich AJ, Barusevicius A, et al. CD117: a sensitive marker for gastrointestinal stromal tumors that is more specific than CD34. Mod Pathol 1998; 11:728-34.

35 Spritz RA, Strunk KM, Lee ST, et al. A YAC contig spanning a cluster of human type III receptor protein kinase genes (PDGFRA-KIT-KDR) in chromosome segment 4q12. Genomics 1994;15:431-6.

36 Miettinen M, Virolainen M, Sarlomo-Rikala M. Gastrointestinal stromal tumors - value of CD34 antigen in their identification and separation from true leiomyomas and schwannomas. Am $\mathcal{F}$ Surg Pathol 1995;19:207-16.

37 Mikhael AI, Bacchi CE, Zarbo RJ, et al. CD34 expression in stromal tumors of the gastrointestinal tract. Appl Immunohistochem 1994;2:89-93.

38 Sueki H, Whitaker D, Buchsbaum M, et al. Novel interactions between dermal dendrocytes and mast cells in human skin. Implications for hemostasis and matrix repair. Lab Invest 1993;69:160-72.

39 Hirota S, Isozaki K, Moriyama Y, et al. Gain-of-function mutations in c-kit in human gastrointestinal stromal mutations in c-kit in human

40 Sandlow JI, Feng HL, Sandra A. Localisation and expression of the c-kit receptor protein in human and expression of the c-kit receptor protein in huma

41 Geissler EN, Ryan MA, Housman DE. The dominant-white spotting (W) locus of the mouse encodes the c-kit

42 Chabot B, Stephenson DA, Chapman VM, et al. The protooncogene c-kit encoding a transmembrane tyrosine kinase receptor maps to the mouse W locus. Nature 1988;335:889.

43 Vliagoftis $\mathrm{H}$, Worobec AS, Metcalfe DD. The protooncogene c-kit and c-kit ligand in human disease. 7 Allergy Clin Immunol 1997;100:435-40.

44 de Saint Aubain Somerhausen N, Fletcher CDM. Gastrointestinal stromal tumours: an update. Sarcoma 1998;2: 131-41.

45 Lammie A, Drobjnak M, Gerald W, et al. Expression of c-kit and kit ligand proteins in normal human tissues. $f$ Histochem Cytochem 1994;42:1417-25.
46 Nakahara $M$, Isozaki $\mathrm{K}$, Hirota $\mathrm{S}$, et al. A novel gain-of-function mutation of c-kit gene in gastrointestinal stromal tumors. Gastroenterology 1998;115:1090-5.

47 Nishida T, Hirota S, Taniguchi M, et al. Familial gastrointestinal stromal tumours with germline mutation of the KIT gene. Nat Genet 1998;19:323-4.

48 Sakurai S, Fukasawa T, Chong JM, et al. C-kit gene bnormalities in gastrointestinal stromal tumors (tumors of interstitial cells of Cajal). Fpn f Cancer Res 1999;90:1321-8.

49 El-Rifai W, Sarlomo-Rikala M, Miettinen M, et al. DNA copy number losses in chromosome 14: an early change in gastrointestinal stromal tumors. Cancer Res 1996;56:3230-3.

50 Sarlomo-Rikala M, El-Rifai W, Andersson L, et al. Different patterns of DNA copy number changes in gastrointestinal stromal tumors, leiomyomas and schwannomas. Hum (1998;29:476-81.

51 El-Rifai W, Sarlomo-Rikala M, Andersson LC, et al. High-resolution deletion mapping of chromosome 14 in stromal tumors of the gastrointestinal tract suggests two distinct tumor suppressor loci. Genes Chromosomes Cancer 2000;27:387-91

52 Lasota J, Jasinski M, Sarlomo-Rikala M, et al. Mutations in exon 11 of c-Kit occur preferentially in malignant versus benign gastrointestinal stromal tumors and do not occur in leiomyomas or leiomyosarcomas. Am f Pathol 1999;154: 53-60.

53 Taniguchi $M$, Nishida T, Hirota S, et al. Effect of c-kit mutation on prognosis of gastrointestinal stromal tumors. Cancer Res 1999;59:4297-300.

54 Hagger R, Finlayson C, Jeffrey I, et al. Role of the interstitial cells of Cajal in the control of gut motility. Br F Surg 1997; 84:445-50.

55 Emory TS, Sobin LH, Lukes L, et al. Prognosis of gastrointestinal smooth-muscle (stromal) tumors. Dependgastrointestinal smooth-muscle (stromal) tumors. Depend-

56 Newman PL, Wadden C, Fletcher CDM. Gastrointestinal stromal tumours: correlation of immunophenotype with clinicopathologic features. F Pathol 1991;164:107-17.

57 Rudolph P, Gloeckner K, Parwaresh R, et al. Immunophenotype, proliferation, DNA ploidy, and biological behavour of gastrointestinal stromal tumors: a multivariate clinicopathologic study. Hum Pathol 1998;29:791-800.

58 Tworek JA, Appelman HD, Singleton TP, et al. Stromal tumors of the jejenum and ileum. Mod Pathol 1997;10:200-

59 Ranchod M, Kempson RL. Smooth muscle tumors of the gastrointestinal tract and retroperitoneum: a pathologic analysis of 100 cases. Cancer 1977;39:255-62.

60 Carillo R, Candia A, Rodriguez-Peralto JL, et al. Prognostic significance of DNA ploidy and proliferative index (MIB-1 index) in gastrointestinal stromal tumors. Hum Pathol 1997;28:160-5.

61 Chou FF, Eng HL, Sheen-Chen SM. Smooth muscle tumors of the gastrointestinal tract: analysis of prognostic factors. Surgery 1996;119:171-7.

62 Cooper PN, Quirke P, Hardy GJ, et al. A flow cytometric, clinical, and histological study of stromal neoplasms of the gastrointestinal tract. Am f Surg Pathol 1992;16:163-70.

63 Cunningham RE, Federspiel BH, McCarthy WF, et al. Predicting prognosis of gastrointestinal smooth muscle tumetry, and image cytometry. Am 7 Surg Pathol 1993;17: $588-94$.

64 El-Naggar AK, Ro JY, McLemore D, et al. Gastrointestinal stromal tumors: DNA flow-cytometric study of 58 patients
with at least five years of follow-up. Mod Pathol 1989;2: 511-15.

65 Emory TS, Derringer GA, Sobin LH, et al. Ki-67 (MIB-1) immunohistochemistry as a prognostic factor in gastrointestinal smooth-muscle tumors. F Surg Pathol 1997;2: $239-42$

66 Evans HL. Smooth muscle tumors of the gastrointestinal tract. A study of 56 cases followed for a minimum of 10 years. Cancer 1985;56:2242-50.

67 Franquement DW. Differentiation and risk assessment of gastrointestinal stromal tumors. Am f Clin Pathol 1995;103: $41-7$.

68 Franquement DW, Frierson HF. Proliferating cell nuclear antigen immunoreactivity and prognosis of gastrointestinal stromal tumors. Mod Pathol 1995;8:473-7.

69 Goldblum JR, Appelman HD. Stromal tumors of the duodenum. A histologic and immunohistochemical study duodenum. A histologic and immunohistoch

70 Haque S, Dean PJ. Stromal neoplasms of the rectum and anal canal. Hum Pathol 1992;23:762-7.

71 Tworek JA, Goldblum JR, Weiss SW, et al. Stromal tumors of the abdominal colon. A clinicopathologic study of 20 cases. Am F Surg Pathol 1999;23:937-45.

72 Tworek JA, Goldblum JR, Weiss SW, et al. Stromal tumors of the anorectum. A clinicopathologic study of 22 cases. Am F Surg Pathol 1999;23:946-54.

73 DeMatteo RP, Lewis JJ, Leung D, et al. Two hundred gastrointestinal stromal tumors: recurrence pattern and prognostic factors for survival. Ann Surg 2000;231:51-8. 